\title{
Palladium-Catalyzed [3 + 3] Cycloaddition of Trimethylenemethane with
} Azomethine Imines

\author{
Ryo Shintani and Tamio Hayashi* \\ Department of Chemistry, Graduate School of Science, Kyoto University, \\ Sakyo, Kyoto 606-8502, Japan
}

\section{Supporting Information}

\section{General}

All air- and moisture-sensitive manipulations were carried out with standard Schlenk techniques under nitrogen or in a glove box under argon.

Toluene and THF were purified by passing through a neutral alumina column under nitrogen. 1,2-Dichloroethane and $\mathrm{CH}_{2} \mathrm{Cl}_{2}$ were distilled over $\mathrm{CaH}_{2}$ under nitrogen. $\mathrm{MeOH}$ was distilled over Mg turnings under nitrogen.

p-Tolualdehyde (Wako Chemicals), $m$-chlorobenzaldehyde (Wako Chemicals), otolualdehyde (TCI), 3-pyridinecarboxaldehyde (Wako Chemicals), pivaldehyde (Aldrich), benzaldehyde (Wako Chemicals), p-trifluoromethylbenzaldehyde (Wako Chemicals), methyl crotonate (TCI), hydrazine monohydrate (Wako Chemicals), triphenylphosphine (Wako Chemicals), and $\mathrm{Pd}(\mathrm{OAc})_{2}$ (Furuya Metal) were used as received.

(2-(Acetoxymethyl)-2-propenyl)trimethylsilane $(\mathbf{1}),{ }^{1} \quad\left(2-\left(1^{\prime}\right.\right.$-acetoxyethyl)-2propenyl)trimethylsilane (4), ${ }^{2}$ (2-(acetoxymethyl)-1-buten-3-yl)trimethylsilane (5), ${ }^{2}$ pyrazolidin-3-one, ${ }^{3}$ 4,4-dimethylpyrazolidin-3-one, ${ }^{3}$ 1-benzylidene-3-oxopyrazolidin1-ium-2-ide (2a), 1-(p-trifluoromethylbenzylidene)-3-oxopyrazolidin-1-ium-2-ide $(2 \mathbf{c}),{ }^{4} \quad 1$-(o - fluorobenzylidene)-3-oxopyrazolidin-1-ium-2-ide $(2 \mathbf{e}),{ }^{4} \quad 1-(1-$ cyclohexenylmethylidene)-3-oxopyrazolidin-1-ium-2-ide $(\mathbf{2 h}){ }^{4}$ 1-benzylidene-4,4dimethyl-3-oxopyrazolidin-1-ium-2-ide $(2 \mathbf{j}),{ }^{4} \mathrm{Pd}\left(\mathrm{PPh}_{3}\right)_{4},{ }^{5}$ and $\mathrm{CpPd}\left(\eta^{3}-\mathrm{C}_{3} \mathrm{H}_{5}\right)^{6}$ were

\footnotetext{
${ }^{1}$ Trost, B. M.; Chan, D. M. T. J. Am. Chem. Soc. 1983, 105, 2315.

2 Trost, B. M.; Chan, D. M. T. J. Am. Chem. Soc. 1981, 103, 5972.

${ }^{3}$ Perri, S. T.; Slater, S. C.; Toske, S. G.; White, J. D. J. Org. Chem. 1990, 55, 6037.

${ }^{4}$ Shintani, R.; Fu, G. C. J. Am. Chem. Soc. 2003, 125, 10778.

${ }^{5}$ Coulson, D. R. Inorg. Synth. 1972, 13, 121.
} 
synthesized following the literature procedures.

All other chemicals and solvents were purchased from Aldrich, Wako Chemicals, TCI, or Kanto Chemicals and used as received.

\section{Synthesis of Substrates}

The yields have not been optimized.

1-(p-Methylbenzylidene)-3-oxopyrazolidin-1-ium-2-ide (2b) (CAS 62516-59-0)<smiles>CCCCCCNC(=O)CCN=Cc1ccc(C)cc1</smiles>

$p$-Tolualdehyde ( $245 \mu \mathrm{L}, 2.08 \mathrm{mmol})$ was added to a solution of pyrazolidin-3-one $(179 \mathrm{mg}, 2.08 \mathrm{mmol})$ in $\mathrm{MeOH}(0.50 \mathrm{~mL})$. The mixture was stirred for $1 \mathrm{~h}$ at room temperature and then diluted with $\mathrm{Et}_{2} \mathrm{O}(2.0 \mathrm{~mL})$. The precipitate was collected by filtration, washed with $\mathrm{Et}_{2} \mathrm{O}$, and dried under vacuum to afford compound $\mathbf{2 b}$ as a pale yellow solid (240 mg, $1.27 \mathrm{mmol} ; 61 \%$ yield).

${ }^{1} \mathrm{H}$ NMR (DMSO- $\left.d_{6}\right): \delta 8.17\left(\mathrm{~d},{ }^{3} J_{\mathrm{HH}}=8.0 \mathrm{~Hz}, 2 \mathrm{H}\right), 7.59(\mathrm{~s}, 1 \mathrm{H}), 7.34\left(\mathrm{~d},{ }^{3} J_{\mathrm{HH}}=8.1\right.$ $\mathrm{Hz}, 2 \mathrm{H}), 4.52\left(\mathrm{t},{ }^{3} \mathrm{~J}_{\mathrm{HH}}=8.0 \mathrm{~Hz}, 2 \mathrm{H}\right), 2.55\left(\mathrm{t},{ }^{3} \mathrm{~J}_{\mathrm{HH}}=8.1 \mathrm{~Hz}, 2 \mathrm{H}\right), 2.36(\mathrm{~s}, 3 \mathrm{H}) .{ }^{13} \mathrm{C} \mathrm{NMR}$ (DMSO- $\left.d_{6}\right): \delta 184.8,141.8,132.9,131.3,129.6,127.4,57.3,29.5,21.4$.

1-(m-Chlorobenzylidene)-3-oxopyrazolidin-1-ium-2-ide (2d) (CAS 61283-27-0)

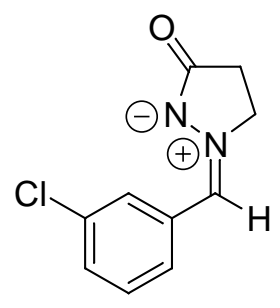

2d

This was synthesized from $m$-chlorobenzaldehyde, following the procedure for compound $\mathbf{2 b}$. White solid, $63 \%$ yield.

${ }^{1} \mathrm{H}$ NMR (DMSO- $\left.d_{6}\right): \delta 8.55(\mathrm{~s}, 1 \mathrm{H}), 8.07-8.05(\mathrm{~m}, 1 \mathrm{H}), 7.66(\mathrm{~s}, 1 \mathrm{H}), 7.58-7.54(\mathrm{~m}$,

\footnotetext{
${ }^{6}$ Parker, G.; Werner, H. Helv. Chim. Acta 1973, 56, 2819.
} 
$2 \mathrm{H}), 4.59\left(\mathrm{t},{ }^{3} \mathrm{~J}_{\mathrm{HH}}=8.0 \mathrm{~Hz}, 2 \mathrm{H}\right), 2.58\left(\mathrm{t},{ }^{3} \mathrm{~J}_{\mathrm{HH}}=8.1 \mathrm{~Hz}, 2 \mathrm{H}\right) .{ }^{13} \mathrm{C}$ NMR $\left(\mathrm{DMSO}-d_{6}\right): \delta$ $184.6,133.3,131.8,130.53,130.46,129.9,129.53,129.46,57.7,29.0$.

1-(o-Methylbenzylidene)-3-oxopyrazolidin-1-ium-2-ide (2f)

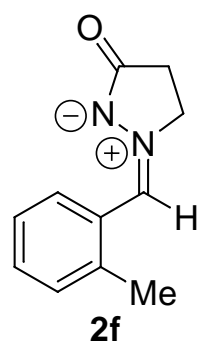

This was synthesized from o-tolualdehyde, following the procedure for compound $\mathbf{2 b}$. White solid, $61 \%$ yield.

${ }^{1} \mathrm{H}$ NMR $\left(\right.$ DMSO- $\left.d_{6}\right): \delta 8.93\left(\mathrm{~d},{ }^{3} J_{\mathrm{HH}}=8.1 \mathrm{~Hz}, 1 \mathrm{H}\right), 7.68(\mathrm{~s}, 1 \mathrm{H}), 7.39-7.31(\mathrm{~m}, 3 \mathrm{H})$, $4.60\left(\mathrm{t},{ }^{3} \mathrm{~J}_{\mathrm{HH}}=8.4 \mathrm{~Hz}, 2 \mathrm{H}\right), 2.56\left(\mathrm{t},{ }^{3} \mathrm{~J}_{\mathrm{HH}}=8.2 \mathrm{~Hz}, 2 \mathrm{H}\right), 2.47(\mathrm{~s}, 3 \mathrm{H}) .{ }^{13} \mathrm{C}$ NMR (DMSO$\left.d_{6}\right): \delta 184.5,138.4,130.8,130.5,130.2,129.4,128.3,126.0,57.8,29.1,19.4$. HRMS (ESI) calcd for $\mathrm{C}_{11} \mathrm{H}_{13} \mathrm{~N}_{2} \mathrm{O}\left(\mathrm{M}+\mathrm{H}^{+}\right)$189.1022, found 189.1030 .

1-(3-Pyridiylmethylidene)-3-oxopyrazolidin-1-ium-2-ide (2g) (CAS 84198-94-7)<smiles>O=C1CCN(Cc2cccnc2)C1</smiles>

This was synthesized from 3-pyridinecarboxaldehyde, following the procedure for compound $\mathbf{2 b}$. Pale yellow solid, 58\% yield.

${ }^{1} \mathrm{H}$ NMR (DMSO- $\left.d_{6}\right): \delta 9.19\left(\mathrm{~d},{ }^{4} \mathrm{~J}_{\mathrm{HH}}=1.9 \mathrm{~Hz}, 1 \mathrm{H}\right), 8.82\left(\mathrm{dt},{ }^{3} \mathrm{~J}_{\mathrm{HH}}=8.0 \mathrm{~Hz}\right.$ and ${ }^{4} \mathrm{~J}_{\mathrm{HH}}$ $=1.9 \mathrm{~Hz}, 1 \mathrm{H}), 8.63\left(\mathrm{dd},{ }^{3} J_{\mathrm{HH}}=4.7 \mathrm{~Hz}\right.$ and $\left.{ }^{4} J_{\mathrm{HH}}=1.7 \mathrm{~Hz}, 1 \mathrm{H}\right), 7.71(\mathrm{~s}, 1 \mathrm{H}), 7.57(\mathrm{dd}$, ${ }^{3} J_{\mathrm{HH}}=8.2$ and $\left.4.7 \mathrm{~Hz}, 1 \mathrm{H}\right), 4.61\left(\mathrm{t},{ }^{3} \mathrm{~J}_{\mathrm{HH}}=8.0 \mathrm{~Hz}, 2 \mathrm{H}\right), 2.59\left(\mathrm{t},{ }^{3} \mathrm{~J}_{\mathrm{HH}}=8.1 \mathrm{~Hz}, 2 \mathrm{H}\right) .{ }^{13} \mathrm{C}$ NMR (DMSO- $\left.d_{6}\right): \delta 184.6,151.4,150.7,137.0,128.7,126.4,123.8,57.7,29.2$.

1-(2,2-Dimethylpropylidene)-3-oxopyrazolidin-1-ium-2-ide (2i) 


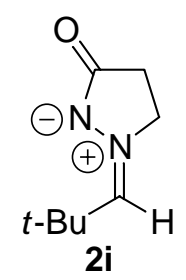

This was synthesized from pivaldehyde, following the procedure for compound 2b. White solid, $57 \%$ yield.

${ }^{1} \mathrm{H}$ NMR (DMSO- $\left.d_{6}\right): \delta 6.77(\mathrm{~s}, 1 \mathrm{H}), 4.31\left(\mathrm{t},{ }^{3} J_{\mathrm{HH}}=8.2 \mathrm{~Hz}, 2 \mathrm{H}\right), 2.43\left(\mathrm{t},{ }^{3} J_{\mathrm{HH}}=8.3\right.$ $\mathrm{Hz}, 2 \mathrm{H}), 1.25(\mathrm{~s}, 9 \mathrm{H}) .{ }^{13} \mathrm{C}$ NMR (DMSO- $\left.d_{6}\right): \delta 182.9,145.2,56.7,33.7,29.5,25.8$. HRMS (ESI) calcd for $\mathrm{C}_{8} \mathrm{H}_{14} \mathrm{~N}_{2} \mathrm{ONa}\left(\mathrm{M}+\mathrm{Na}^{+}\right)$177.0998, found 177.1007.

\section{5-Methylpyrazolidin-3-one (CAS 10234-76-1)}

This was synthesized from methyl crotonate and hydrazine monohydrate, following the procedure for pyrazolidin-3-one. ${ }^{3}$ Pale yellow oil, $100 \%$ yield.

${ }^{1} \mathrm{H}$ NMR $\left(\mathrm{CDCl}_{3}\right): \delta 3.83-3.76(\mathrm{~m}, 1 \mathrm{H}), 2.55\left(\mathrm{dd},{ }^{2} J_{\mathrm{HH}}=16.2 \mathrm{~Hz}\right.$ and ${ }^{3} J_{\mathrm{HH}}=7.1 \mathrm{~Hz}$, $1 \mathrm{H}), 2.18\left(\mathrm{dd},{ }^{2} J_{\mathrm{HH}}=16.2 \mathrm{~Hz}\right.$ and $\left.{ }^{3} J_{\mathrm{HH}}=8.8 \mathrm{~Hz}, 1 \mathrm{H}\right), 1.29\left(\mathrm{~d},{ }^{3} J_{\mathrm{HH}}=6.3 \mathrm{~Hz}, 3 \mathrm{H}\right)$.

1-Benzylidene-5-methyl-3-oxopyrazolidin-1-ium-2-ide (2k) (CAS 14893-83-5)<smiles>CC1CC(=O)N(Cl)C1=Cc1ccccc1</smiles>

This was synthesized from benzaldehyde and 5-methylpyrazolidin-3-one, following the procedure for compound $\mathbf{2 b}$. White solid, $62 \%$ yield.

${ }^{1} \mathrm{H}$ NMR (DMSO- $\left.d_{6}\right): \delta \quad 8.32-8.30(\mathrm{~m}, 2 \mathrm{H}), 7.72(\mathrm{~s}, 1 \mathrm{H}), 7.55-7.50(\mathrm{~m}, 3 \mathrm{H}), 4.84-4.78$ $(\mathrm{m}, 1 \mathrm{H}), 2.84\left(\mathrm{dd},{ }^{2} J_{\mathrm{HH}}=16.3 \mathrm{~Hz}\right.$ and $\left.{ }^{3} J_{\mathrm{HH}}=9.1 \mathrm{~Hz}, 1 \mathrm{H}\right), 2.24\left(\mathrm{dd},{ }^{2} J_{\mathrm{HH}}=16.3 \mathrm{~Hz}\right.$ and $\left.{ }^{3} J_{\mathrm{HH}}=4.1 \mathrm{~Hz}, 1 \mathrm{H}\right), 1.55\left(\mathrm{~d},{ }^{3} J_{\mathrm{HH}}=6.7 \mathrm{~Hz}, 3 \mathrm{H}\right) .{ }^{13} \mathrm{C}$ NMR $\left(\mathrm{DMSO}-d_{6}\right): \delta 183.2,132.0$, 131.4, 131.2, 130.1, 128.8, 65.7, 37.2, 22.2.

$N$-( $p$-Ethoxycarbonylphenyl)- $\alpha$-( $p$-trifluoromethylphenyl)nitrone (8) 
<smiles>CCOC(=O)c1ccc([N+]([O-])=Cc2ccc(C(F)(F)F)cc2)cc1</smiles>

p-Trifluoromethylbenzaldehyde $(290 \mu \mathrm{L}, 2.12 \mathrm{mmol})$ was added to a solution of ethyl $p$-hydroxylaminobenzoate $(385 \mathrm{mg}, 2.12 \mathrm{mmol})$ in $\mathrm{EtOH}(1.5 \mathrm{~mL})$. The mixture was stirred for $2 \mathrm{~h}$ at room temperature and then diluted with $\mathrm{MeOH}$. The precipitate was collected by filtration, washed with $\mathrm{MeOH}$, and dried under vacuum to afford compound 8 as a white solid (185 mg, $0.55 \mathrm{mmol} ; 26 \%$ yield).

${ }^{1} \mathrm{H}$ NMR $\left(\mathrm{C}_{6} \mathrm{D}_{6}\right): \delta 8.23\left(\mathrm{~d},{ }^{3} J_{\mathrm{HH}}=8.0 \mathrm{~Hz}, 2 \mathrm{H}\right), 8.01\left(\mathrm{~d},{ }^{3} J_{\mathrm{HH}}=8.7 \mathrm{~Hz}, 2 \mathrm{H}\right), 7.39(\mathrm{~d}$, $\left.{ }^{3} \mathrm{~J}_{\mathrm{HH}}=8.0 \mathrm{~Hz}, 2 \mathrm{H}\right), 7.38\left(\mathrm{~d},{ }^{3} \mathrm{~J}_{\mathrm{HH}}=8.5 \mathrm{~Hz}, 2 \mathrm{H}\right), 7.12(\mathrm{~s}, 1 \mathrm{H}), 4.11\left(\mathrm{q},{ }^{3} \mathrm{~J}_{\mathrm{HH}}=7.1 \mathrm{~Hz}, 2 \mathrm{H}\right)$, $1.01\left(\mathrm{t},{ }^{3} \mathrm{~J}_{\mathrm{HH}}=7.1 \mathrm{~Hz}, 3 \mathrm{H}\right) .{ }^{13} \mathrm{C}$ NMR $\left(\mathrm{DMSO}-d_{6}\right): \delta 164.7,151.2,134.4,133.6,131.3$, $130.1,130.0\left(q^{2}{ }^{2} J_{\mathrm{CF}}=32.1 \mathrm{~Hz}\right), 129.4,125.4\left(\mathrm{q},{ }^{3} J_{\mathrm{CF}}=4.1 \mathrm{~Hz}\right), 123.9\left(\mathrm{q},{ }^{1} J_{\mathrm{CF}}=272 \mathrm{~Hz}\right)$, 122.1, 61.2, 14.1. HRMS (ESI) calcd for $\mathrm{C}_{17} \mathrm{H}_{15} \mathrm{~F}_{3} \mathrm{NO}_{3}\left(\mathrm{M}+\mathrm{H}^{+}\right)$338.0999, found 338.1007 .

\section{Catalytic Reactions}

\section{General Procedure for Table 2 and Equations 2-3.}

A solution of $\mathrm{Pd}\left(\mathrm{PPh}_{3}\right)_{4}(18.5 \mathrm{mg}, 16.0 \mu \mathrm{mol})$, (2-(acetoxymethyl)-2propenyl)trimethylsilane 1 (74.5 $\mathrm{mg}, 0.400 \mathrm{mmol})$, and azomethine imine 2 (0.200 mmol) in $\mathrm{CH}_{2} \mathrm{Cl}_{2}(1.0 \mathrm{~mL})$ was stirred for $48 \mathrm{~h}$ at $40{ }^{\circ} \mathrm{C}$, and the reaction mixture was directly passed through a pad of silica gel with EtOAc. After removing the solvent under vacuum, the residue was purified by silica gel preparative TLC to afford compound 3.

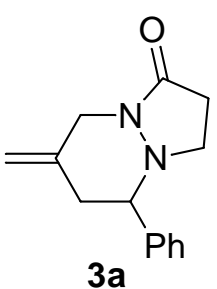

Entry 1. White solid. $81 \%$ yield.

${ }^{1} \mathrm{H}$ NMR $\left(\mathrm{CDCl}_{3}\right): \delta 7.39-7.30(\mathrm{~m}, 5 \mathrm{H}), 5.01(\mathrm{~s}, 1 \mathrm{H}), 4.89(\mathrm{~s}, 1 \mathrm{H}), 4.59\left(\mathrm{~d},{ }^{2} J_{\mathrm{HH}}=13.8\right.$ 
$\mathrm{Hz}, 1 \mathrm{H}), 3.65\left(\mathrm{~d},{ }^{2} J_{\mathrm{HH}}=13.5 \mathrm{~Hz}, 1 \mathrm{H}\right), 3.36\left(\mathrm{dd},{ }^{2} J_{\mathrm{HH}}=11.3 \mathrm{~Hz}\right.$ and $\left.{ }^{3} J_{\mathrm{HH}}=2.8 \mathrm{~Hz}, 1 \mathrm{H}\right)$, $3.21\left(\mathrm{td}, J_{\mathrm{HH}}=10.1 \mathrm{~Hz}\right.$ and $\left.{ }^{3} \mathrm{~J}_{\mathrm{HH}}=5.0 \mathrm{~Hz}, 1 \mathrm{H}\right), 2.66\left(\mathrm{q}, J_{\mathrm{HH}}=9.6 \mathrm{~Hz}, 1 \mathrm{H}\right), 2.59-2.38(\mathrm{~m}$, $4 \mathrm{H}) .{ }^{13} \mathrm{C} \mathrm{NMR}\left(\mathrm{CDCl}_{3}\right): \delta 170.0,140.2,138.7,129.0,128.5,127.7,111.9,71.6,48.5,47.6$, 42.4, 30.7. Anal. Calcd for $\mathrm{C}_{14} \mathrm{H}_{16} \mathrm{~N}_{2} \mathrm{O}$ : C, 73.66; $\mathrm{H}, 7.06$. Found: $\mathrm{C}, 73.54 ; \mathrm{H}, 7.26$.

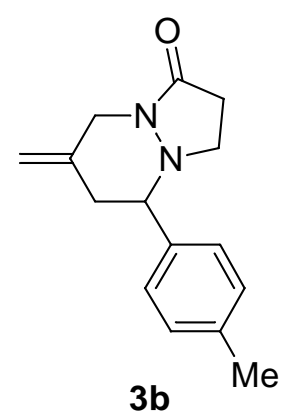

Entry 2. Colorless oil. $74 \%$ yield.

${ }^{1} \mathrm{H}$ NMR $\left(\mathrm{CDCl}_{3}\right): \delta 7.25\left(\mathrm{~d},{ }^{3} \mathrm{~J}_{\mathrm{HH}}=7.8 \mathrm{~Hz}, 2 \mathrm{H}\right), 7.17\left(\mathrm{~d},{ }^{3} \mathrm{~J}_{\mathrm{HH}}=7.9 \mathrm{~Hz}, 2 \mathrm{H}\right), 5.00(\mathrm{~s}$, $1 \mathrm{H}), 4.88(\mathrm{~s}, 1 \mathrm{H}), 4.59\left(\mathrm{~d},{ }^{2} J_{\mathrm{HH}}=14.2 \mathrm{~Hz}, 1 \mathrm{H}\right), 3.65\left(\mathrm{~d},{ }^{2} J_{\mathrm{HH}}=14.4 \mathrm{~Hz}, 1 \mathrm{H}\right), 3.32\left(\mathrm{~d},{ }^{2} J_{\mathrm{HH}}\right.$ $=11.0 \mathrm{~Hz}, 1 \mathrm{H}), 3.20\left(\mathrm{td}, J_{\mathrm{HH}}=10.1 \mathrm{~Hz}\right.$ and $\left.{ }^{3} J_{\mathrm{HH}}=5.0 \mathrm{~Hz}, 1 \mathrm{H}\right), 2.66\left(\mathrm{q}, J_{\mathrm{HH}}=9.5 \mathrm{~Hz}\right.$, $1 \mathrm{H}), 2.59-2.51(\mathrm{~m}, 2 \mathrm{H}), 2.48-2.34(\mathrm{~m}, 2 \mathrm{H}), 2.36(\mathrm{~s}, 3 \mathrm{H}) .{ }^{13} \mathrm{C} \mathrm{NMR}\left(\mathrm{CDCl}_{3}\right): \delta$ 170.0, $138.8,138.2,137.1,129.7,127.5,111.8,71.3,48.4,47.5,42.4,30.6,21.3$. Anal. Calcd for $\mathrm{C}_{15} \mathrm{H}_{18} \mathrm{~N}_{2} \mathrm{O}: \mathrm{C}, 74.35 ; \mathrm{H}, 7.49$. Found: $\mathrm{C}, 74.19 ; \mathrm{H}, 7.50$.

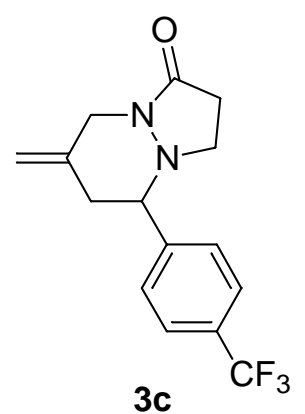

Entry 3. White solid. 92\% yield.

${ }^{1} \mathrm{H} \mathrm{NMR}\left(\mathrm{CDCl}_{3}\right): \delta 7.64\left(\mathrm{~d},{ }^{3} J_{\mathrm{HH}}=8.3 \mathrm{~Hz}, 2 \mathrm{H}\right), 7.51\left(\mathrm{~d},{ }^{3} \mathrm{~J}_{\mathrm{HH}}=8.0 \mathrm{~Hz}, 2 \mathrm{H}\right), 5.04(\mathrm{~s}$, $1 \mathrm{H}), 4.91(\mathrm{~s}, 1 \mathrm{H}), 4.61\left(\mathrm{~d},{ }^{2} J_{\mathrm{HH}}=13.9 \mathrm{~Hz}, 1 \mathrm{H}\right), 3.65\left(\mathrm{~d},{ }^{2} J_{\mathrm{HH}}=13.9 \mathrm{~Hz}, 1 \mathrm{H}\right), 3.44(\mathrm{dd}$, ${ }^{2} J_{\mathrm{HH}}=10.7 \mathrm{~Hz}$ and $\left.{ }^{3} J_{\mathrm{HH}}=3.7 \mathrm{~Hz}, 1 \mathrm{H}\right), 3.24\left(\mathrm{td}, J_{\mathrm{HH}}=10.2 \mathrm{~Hz}\right.$ and $\left.{ }^{3} J_{\mathrm{HH}}=4.8 \mathrm{~Hz}, 1 \mathrm{H}\right)$, 2.66-2.42 (m, 5H). ${ }^{13} \mathrm{C} \mathrm{NMR}\left(\mathrm{CDCl}_{3}\right): \delta 169.9,144.3,138.0,130.7\left(\mathrm{q},{ }^{2} J_{\mathrm{CF}}=32.6 \mathrm{~Hz}\right)$, 128.0, $126.0\left(\mathrm{q}^{3} \mathrm{~J}_{\mathrm{CF}}=4.1 \mathrm{~Hz}\right), 124.1\left(\mathrm{q},{ }^{1} J_{\mathrm{CF}}=271.7 \mathrm{~Hz}\right), 112.3,71.1,48.6,47.5,42.4$, 30.6. Anal. Calcd for $\mathrm{C}_{15} \mathrm{H}_{15} \mathrm{~F}_{3} \mathrm{~N}_{2} \mathrm{O}$ : $\mathrm{C}, 60.81 ; \mathrm{H}, 5.10$. Found: $\mathrm{C}, 60.73 ; \mathrm{H}, 5.40$. 


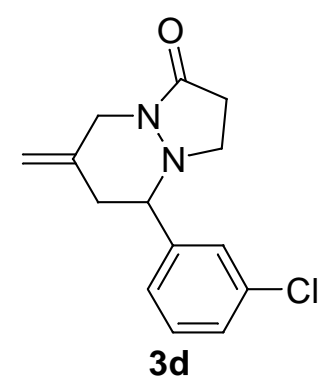

Entry 4. Colorless oil. $90 \%$ yield.

${ }^{1} \mathrm{H}$ NMR $\left(\mathrm{CDCl}_{3}\right): \delta 7.39(\mathrm{~s}, 1 \mathrm{H}), 7.31-7.29(\mathrm{~m}, 2 \mathrm{H}), 7.27-7.24(\mathrm{~m}, 1 \mathrm{H}), 5.02(\mathrm{~s}, 1 \mathrm{H})$, $4.90(\mathrm{~s}, 1 \mathrm{H}), 4.59\left(\mathrm{~d},{ }^{2} J_{\mathrm{HH}}=13.8 \mathrm{~Hz}, 1 \mathrm{H}\right), 3.63\left(\mathrm{~d},{ }^{2} J_{\mathrm{HH}}=13.8 \mathrm{~Hz}, 1 \mathrm{H}\right), 3.34\left(\mathrm{dd},{ }^{2} J_{\mathrm{HH}}=\right.$ $10.8 \mathrm{~Hz}$ and $\left.{ }^{3} J_{\mathrm{HH}}=3.9 \mathrm{~Hz}, 1 \mathrm{H}\right), 3.25\left(\mathrm{td}, J_{\mathrm{HH}}=10.0 \mathrm{~Hz}\right.$ and $\left.{ }^{3} J_{\mathrm{HH}}=5.0 \mathrm{~Hz}, 1 \mathrm{H}\right), 2.65(\mathrm{q}$, $\left.J_{\mathrm{HH}}=9.3 \mathrm{~Hz}, 1 \mathrm{H}\right), 2.59-2.40(\mathrm{~m}, 4 \mathrm{H}) .{ }^{13} \mathrm{C} \mathrm{NMR}\left(\mathrm{CDCl}_{3}\right): \delta 169.9,142.3,138.1,134.9$, 130.3, 128.6, 127.7, 125.8, 112.1, 70.9, 48.6, 47.5, 42.3, 30.6. Anal. Calcd for $\mathrm{C}_{14} \mathrm{H}_{15} \mathrm{ClN}_{2} \mathrm{O}: \mathrm{C}, 64.00 ; \mathrm{H}, 5.75$. Found: $\mathrm{C}, 63.71 ; \mathrm{H}, 5.80$.

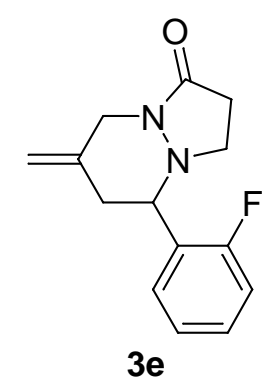

Entry 5. Pale yellow oil. $88 \%$ yield.

${ }^{1} \mathrm{H}$ NMR $\left(\mathrm{CDCl}_{3}\right): \delta 7.54\left(\mathrm{t},{ }^{3} J_{\mathrm{HH}}=7.3 \mathrm{~Hz}, 1 \mathrm{H}\right), 7.32-7.27(\mathrm{~m}, 1 \mathrm{H}), 7.18\left(\mathrm{t},{ }^{3} J_{\mathrm{HH}}=7.5\right.$ $\mathrm{Hz}, 1 \mathrm{H}), 7.07\left(\mathrm{t},{ }^{3} \mathrm{~J}=9.2 \mathrm{~Hz}, 1 \mathrm{H}\right), 5.03(\mathrm{~s}, 1 \mathrm{H}), 4.92(\mathrm{~s}, 1 \mathrm{H}), 4.61\left(\mathrm{~d},{ }^{2} J_{\mathrm{HH}}=14.0 \mathrm{~Hz}, 1 \mathrm{H}\right)$, $3.85\left(\mathrm{~d},{ }^{2} J_{\mathrm{HH}}=10.3 \mathrm{~Hz}, 1 \mathrm{H}\right), 3.66\left(\mathrm{~d},{ }^{2} J_{\mathrm{HH}}=13.3 \mathrm{~Hz}, 1 \mathrm{H}\right), 3.27\left(\mathrm{td}, J_{\mathrm{HH}}=9.9 \mathrm{~Hz}\right.$ and $\left.{ }^{3} J_{\mathrm{HH}}=5.4 \mathrm{~Hz}, 1 \mathrm{H}\right), 2.70\left(\mathrm{q}, J_{\mathrm{HH}}=9.4 \mathrm{~Hz}, 1 \mathrm{H}\right), 2.62-2.39(\mathrm{~m}, 4 \mathrm{H}) .{ }^{13} \mathrm{C} \mathrm{NMR}\left(\mathrm{CDCl}_{3}\right)$ : $\delta 170.0,160.5\left(\mathrm{~d},{ }^{1} J_{\mathrm{CF}}=246.5 \mathrm{~Hz}\right), 138.2,129.6\left(\mathrm{~d},{ }^{3} J_{\mathrm{CF}}=8.3 \mathrm{~Hz}\right), 128.6,126.9\left(\mathrm{~d},{ }^{2} J_{\mathrm{CF}}=\right.$ $13.0 \mathrm{~Hz}), 124.9\left(\mathrm{~d},{ }^{3} J_{\mathrm{CF}}=3.0 \mathrm{~Hz}\right), 115.8\left(\mathrm{~d},{ }^{2} J_{\mathrm{CF}}=22.2 \mathrm{~Hz}\right), 112.1,62.9,48.3,47.5,40.8$, 30.5. Anal. Calcd for $\mathrm{C}_{14} \mathrm{H}_{15} \mathrm{FN}_{2} \mathrm{O}: \mathrm{C}, 68.28 ; \mathrm{H}, 6.14$. Found: $\mathrm{C}, 68.07 ; \mathrm{H}, 6.16$. 


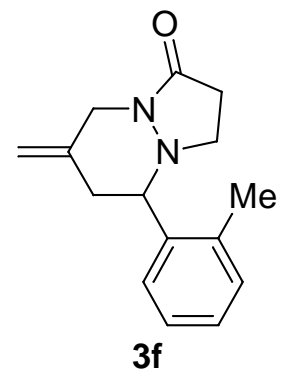

Entry 6. Colorless oil. 70\% yield.

${ }^{1} \mathrm{H} \mathrm{NMR}\left(\mathrm{CDCl}_{3}\right): \delta 7.52(\mathrm{bs}, 1 \mathrm{H}), 7.23\left(\mathrm{t},{ }^{3} J_{\mathrm{HH}}=7.3 \mathrm{~Hz}, 1 \mathrm{H}\right), 7.20-7.15(\mathrm{~m}, 2 \mathrm{H}), 5.00$ $(\mathrm{s}, 1 \mathrm{H}), 4.89(\mathrm{~s}, 1 \mathrm{H}), 4.61\left(\mathrm{~d},{ }^{2} J_{\mathrm{HH}}=14.0 \mathrm{~Hz}, 1 \mathrm{H}\right), 3.68-3.64(\mathrm{~m}, 2 \mathrm{H}), 3.31-3.26(\mathrm{~m}, 1 \mathrm{H})$, 2.62-2.52 (m, 2H), 2.48-2.39 (m, 3H), $2.35(\mathrm{~s}, 3 \mathrm{H}) .{ }^{13} \mathrm{C} \mathrm{NMR}\left(\mathrm{CDCl}_{3}\right): \delta 170.0,138.8$, 138.3, 135.4, 130.8, 127.6, 126.8, 111.7, 66.6, 48.2, 47.5, 41.4, 30.7, 19.7. Anal. Calcd for $\mathrm{C}_{15} \mathrm{H}_{18} \mathrm{~N}_{2} \mathrm{O}: \mathrm{C}, 74.35 ; \mathrm{H}, 7.49$. Found: $\mathrm{C}, 74.10 ; \mathrm{H}, 7.51$.

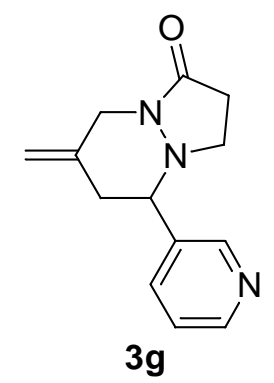

Entry 7. Pale yellow oil. $75 \%$ yield.

${ }^{1} \mathrm{H} \mathrm{NMR}\left(\mathrm{CDCl}_{3}\right): \delta 8.62(\mathrm{~s}, 1 \mathrm{H}), 8.60\left(\mathrm{~d},{ }^{3} J_{\mathrm{HH}}=4.6 \mathrm{~Hz}, 1 \mathrm{H}\right), 7.77\left(\mathrm{~d},{ }^{3} J_{\mathrm{HH}}=7.7 \mathrm{~Hz}\right.$, $1 \mathrm{H}), 7.35\left(\mathrm{dd},{ }^{3} J_{\mathrm{HH}}=7.8\right.$ and $\left.4.7 \mathrm{~Hz}, 1 \mathrm{H}\right), 5.05(\mathrm{~s}, 1 \mathrm{H}), 4.92(\mathrm{~s}, 1 \mathrm{H}), 4.61\left(\mathrm{~d},{ }^{2} J_{\mathrm{HH}}=13.8\right.$ $\mathrm{Hz}, 1 \mathrm{H}), 3.65\left(\mathrm{~d},{ }^{2} J_{\mathrm{HH}}=13.4 \mathrm{~Hz}, 1 \mathrm{H}\right), 3.43\left(\mathrm{~d},{ }^{2} J_{\mathrm{HH}}=8.8 \mathrm{~Hz}, 1 \mathrm{H}\right), 3.22\left(\mathrm{td}, J_{\mathrm{HH}}=10.0\right.$ $\mathrm{Hz}$ and $\left.{ }^{3} \mathrm{~J}_{\mathrm{HH}}=4.7 \mathrm{~Hz}, 1 \mathrm{H}\right), 2.67-2.41(\mathrm{~m}, 5 \mathrm{H}) .{ }^{13} \mathrm{C} \mathrm{NMR}\left(\mathrm{CDCl}_{3}\right): \delta 169.9,150.0,149.3$, $137.8,135.8,135.2,124.1,112.4,69.0,48.7,47.5,42.2$, 30.6. HRMS (ESI) calcd for $\mathrm{C}_{13} \mathrm{H}_{16} \mathrm{~N}_{3} \mathrm{O}\left(\mathrm{M}+\mathrm{H}^{+}\right)$230.1288, found 230.1299.

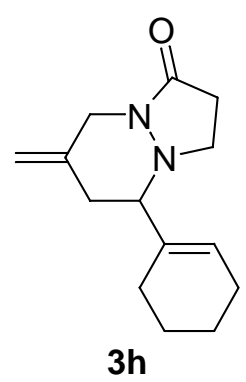

Entry 8 . White solid. $71 \%$ yield. 
${ }^{1} \mathrm{H} \mathrm{NMR}\left(\mathrm{CDCl}_{3}\right): \delta 5.70(\mathrm{~s}, 1 \mathrm{H}), 4.93(\mathrm{~s}, 1 \mathrm{H}), 4.83(\mathrm{~s}, 1 \mathrm{H}), 4.48\left(\mathrm{~d},{ }^{2} J_{\mathrm{HH}}=12.5 \mathrm{~Hz}\right.$, $1 \mathrm{H}), 3.48\left(\mathrm{~d},{ }^{2} J_{\mathrm{HH}}=13.8 \mathrm{~Hz}, 1 \mathrm{H}\right), 3.32\left(\mathrm{td}, J_{\mathrm{HH}}=10.0 \mathrm{~Hz}\right.$ and $\left.{ }^{3} J_{\mathrm{HH}}=5.2 \mathrm{~Hz}, 1 \mathrm{H}\right), 2.78$ $\left(\mathrm{q}, J_{\mathrm{HH}}=9.6 \mathrm{~Hz}, 1 \mathrm{H}\right), 2.72\left(\mathrm{dd},{ }^{2} J_{\mathrm{HH}}=11.7 \mathrm{~Hz}\right.$ and $\left.{ }^{3} J_{\mathrm{HH}}=2.5 \mathrm{~Hz}, 1 \mathrm{H}\right), 2.54\left(\mathrm{ddd},{ }^{2} J_{\mathrm{HH}}=\right.$ $16.6 \mathrm{~Hz}$ and ${ }^{3} \mathrm{~J}_{\mathrm{HH}}=9.0$ and $\left.5.1 \mathrm{~Hz}, 1 \mathrm{H}\right), 2.50-2.37(\mathrm{~m}, 2 \mathrm{H}), 2.24\left(\mathrm{~d},{ }^{2} J_{\mathrm{HH}}=13.6 \mathrm{~Hz}, 1 \mathrm{H}\right)$, 2.06-1.94 (m, 4H), 1.70-1.50 (m, 4H). ${ }^{13} \mathrm{C} \mathrm{NMR}\left(\mathrm{CDCl}_{3}\right): \delta 169.8,139.2,136.5,126.9$, $111.5,73.8,47.8,47.3,38.4,30.7,25.3,24.2,22.9$, 22.7. Anal. Calcd for $\mathrm{C}_{14} \mathrm{H}_{20} \mathrm{~N}_{2} \mathrm{O}$ : C, 72.38; H, 8.68. Found: C, 72.27; H, 8.79.

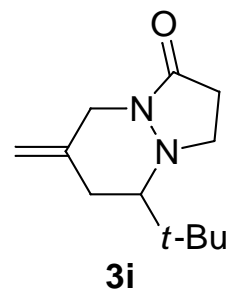

Entry 9. Pale yellow oil. 20\% yield.

${ }^{1} \mathrm{H} \mathrm{NMR}\left(\mathrm{CDCl}_{3}\right): \delta 4.91(\mathrm{~s}, 1 \mathrm{H}), 4.88(\mathrm{~s}, 1 \mathrm{H}), 4.56\left(\mathrm{~d},{ }^{2} J_{\mathrm{HH}}=14.6 \mathrm{~Hz}, 1 \mathrm{H}\right), 3.66(\mathrm{td}$, $J_{\mathrm{HH}}=9.6 \mathrm{~Hz}$ and $\left.{ }^{3} J_{\mathrm{HH}}=3.5 \mathrm{~Hz}, 1 \mathrm{H}\right), 3.50\left(\mathrm{~d},{ }^{2} J_{\mathrm{HH}}=14.4 \mathrm{~Hz}, 1 \mathrm{H}\right), 2.85\left(\mathrm{q}, J_{\mathrm{HH}}=9.8 \mathrm{~Hz}\right.$, $1 \mathrm{H}), 2.59\left(\mathrm{ddd},{ }^{2} J_{\mathrm{HH}}=16.6 \mathrm{~Hz}\right.$ and ${ }^{3} J_{\mathrm{HH}}=8.8$ and $\left.3.6 \mathrm{~Hz}, 1 \mathrm{H}\right), 2.52-2.43(\mathrm{~m}, 2 \mathrm{H}), 2.33$ $\left(\mathrm{dd},{ }^{3} J_{\mathrm{HH}}=8.6\right.$ and $\left.4.1 \mathrm{~Hz}, 1 \mathrm{H}\right), 2.26\left(\mathrm{dd},{ }^{2} J_{\mathrm{HH}}=13.5 \mathrm{~Hz}\right.$ and $\left.{ }^{3} J_{\mathrm{HH}}=9.0 \mathrm{~Hz}, 1 \mathrm{H}\right), 1.02$ (s, 9H). ${ }^{13} \mathrm{C} \mathrm{NMR}\left(\mathrm{CDCl}_{3}\right): \delta 169.5,139.4,110.9,74.0,53.0,47.2,34.9,34.0,31.4,28.5$. HRMS (ESI) calcd for $\mathrm{C}_{12} \mathrm{H}_{21} \mathrm{~N}_{2} \mathrm{O}\left(\mathrm{M}+\mathrm{H}^{+}\right)$209.1648, found 209.1658.

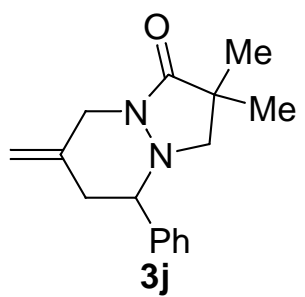

Equation 2. White solid. 94\% yield.

${ }^{1} \mathrm{H} \mathrm{NMR}\left(\mathrm{CDCl}_{3}\right): \delta 7.39-7.30(\mathrm{~m}, 5 \mathrm{H}), 5.02\left(\mathrm{~d},{ }^{2} J_{\mathrm{HH}}=1.5 \mathrm{~Hz}, 1 \mathrm{H}\right), 4.90\left(\mathrm{~d},{ }^{2} J_{\mathrm{HH}}=\right.$ $1.3 \mathrm{~Hz}, 1 \mathrm{H}), 4.57\left(\mathrm{dd},{ }^{2} J_{\mathrm{HH}}=13.9 \mathrm{~Hz}\right.$ and $\left.{ }^{4} J_{\mathrm{HH}}=1.4 \mathrm{~Hz}, 1 \mathrm{H}\right), 3.63\left(\mathrm{~d},{ }^{2} J_{\mathrm{HH}}=13.8 \mathrm{~Hz}\right.$, $1 \mathrm{H}), 3.24\left(\mathrm{dd},{ }^{3} \mathrm{~J}_{\mathrm{HH}}=12.4 \mathrm{~Hz}\right.$ and $\left.2.9 \mathrm{~Hz}, 1 \mathrm{H}\right), 2.97\left(\mathrm{~d},{ }^{2} J_{\mathrm{HH}}=9.7 \mathrm{~Hz}, 1 \mathrm{H}\right), 2.56(\mathrm{dd}$, ${ }^{2} J_{\mathrm{HH}}=13.1 \mathrm{~Hz}$ and $\left.{ }^{3} J_{\mathrm{HH}}=12.4 \mathrm{~Hz}, 1 \mathrm{H}\right), 2.47\left(\mathrm{~d},{ }^{2} J_{\mathrm{HH}}=13.5 \mathrm{~Hz}, 1 \mathrm{H}\right), 2.35\left(\mathrm{~d},{ }^{2} J_{\mathrm{HH}}=9.8\right.$ $\mathrm{Hz}, 1 \mathrm{H}), 1.17(\mathrm{~s}, 3 \mathrm{H}), 1.14(\mathrm{~s}, 3 \mathrm{H}) .{ }^{13} \mathrm{C} \mathrm{NMR}\left(\mathrm{CDCl}_{3}\right): \delta 174.5,140.1,138.8,129.0,128.3$, 127.7, 111.8, 72.4, 63.7, 48.0, 42.6, 41.2, 23.5, 23.4. Anal. Calcd for $\mathrm{C}_{16} \mathrm{H}_{20} \mathrm{~N}_{2} \mathrm{O}$ : C, 74.97; 
H, 7.86. Found: C, 74.77; H, 7.80.

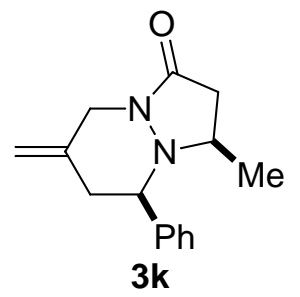

Equation 3. White solid. $87 \%$ yield, $\mathrm{dr}=96 / 4$. Recrystallization from $\mathrm{Et}_{2} \mathrm{O}$ afforded single crystals suitable for $\mathrm{X}$-ray analysis, and the relative configuration of the major diastereomer was determined to be syn.

Major diastereomer: ${ }^{1} \mathrm{H}$ NMR $\left(\mathrm{CDCl}_{3}\right)$ : $\delta$ 7.40-7.30 (m, 5H), $4.97\left(\mathrm{~d},{ }^{2} J_{\mathrm{HH}}=1.5 \mathrm{~Hz}\right.$, $1 \mathrm{H}), 4.83\left(\mathrm{~d},{ }^{2} J_{\mathrm{HH}}=1.3 \mathrm{~Hz}, 1 \mathrm{H}\right), 4.68\left(\mathrm{dd},{ }^{2} J_{\mathrm{HH}}=14.0 \mathrm{~Hz}\right.$ and $\left.{ }^{4} J_{\mathrm{HH}}=1.4 \mathrm{~Hz}, 1 \mathrm{H}\right), 3.68$ $\left(\mathrm{d},{ }^{2} J_{\mathrm{HH}}=13.9 \mathrm{~Hz}, 1 \mathrm{H}\right), 3.52\left(\mathrm{dd},{ }^{3} J_{\mathrm{HH}}=11.4\right.$ and $\left.3.1 \mathrm{~Hz}, 1 \mathrm{H}\right), 3.16\left(\mathrm{dqd},{ }^{3} J_{\mathrm{HH}}=9.0,6.7\right.$, and $3.4 \mathrm{~Hz}, 1 \mathrm{H}), 2.85\left(\mathrm{~d},{ }^{2} J_{\mathrm{HH}}=16.9 \mathrm{~Hz}\right.$ and $\left.{ }^{3} J_{\mathrm{HH}}=8.8 \mathrm{~Hz}, 1 \mathrm{H}\right), 2.63-2.57(\mathrm{~m}, 1 \mathrm{H}), 2.47$ $\left(\mathrm{dt},{ }^{2} J_{\mathrm{HH}}=13.8 \mathrm{~Hz}\right.$ and $\left.J_{\mathrm{HH}}=2.4 \mathrm{~Hz}, 1 \mathrm{H}\right), 2.03\left(\mathrm{ddd},{ }^{2} J_{\mathrm{HH}}=16.9 \mathrm{~Hz}\right.$ and ${ }^{3} J_{\mathrm{HH}}=3.3 \mathrm{~Hz}$ and $\left.{ }^{4} J_{\mathrm{HH}}=1.3 \mathrm{~Hz}, 1 \mathrm{H}\right), 0.98\left(\mathrm{~d},{ }^{3} J_{\mathrm{HH}}=6.6 \mathrm{~Hz}, 3 \mathrm{H}\right) .{ }^{13} \mathrm{C} \mathrm{NMR}\left(\mathrm{CDCl}_{3}\right): \delta$ 169.0, 141.0, 139.1, 129.0, 128.4, 127.6, 111.3, 70.5, 52.8, 46.8, 42.9, 36.8, 22.0. Anal. Calcd for $\mathrm{C}_{15} \mathrm{H}_{18} \mathrm{~N}_{2} \mathrm{O}: \mathrm{C}, 74.35 ; \mathrm{H}, 7.49$. Found: C, 74.11; H, 7.49.

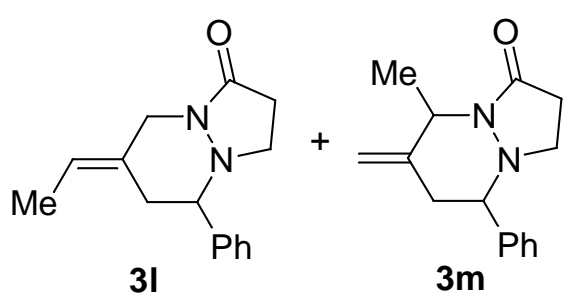

Procedure for Equation 4.

A solution of $\mathrm{Pd}\left(\mathrm{PPh}_{3}\right)_{4}(18.5 \mathrm{mg}, 16.0 \mu \mathrm{mol}),\left(2-\left(1^{\prime}\right.\right.$-acetoxyethyl)-2propenyl)trimethylsilane $4(80.1 \mathrm{mg}, 0.400 \mathrm{mmol})$, and azomethine imine $2 \mathrm{a}(34.8$ $\mathrm{mg}, 0.200 \mathrm{mmol})$ in $\mathrm{CH}_{2} \mathrm{Cl}_{2}(1.0 \mathrm{~mL})$ was stirred for $48 \mathrm{~h}$ at $40{ }^{\circ} \mathrm{C}$, and the reaction mixture was directly passed through a pad of silica gel with EtOAc. After removing the solvent under vacuum, the residue was purified by silica gel preparative TLC with EtOAc/hexane $=1 / 1$ to afford compound 31 as a colorless oil $(27.6 \mathrm{mg}, 0.114$ $\mathrm{mmol} ; 57 \%$ yield) and compound $3 \mathrm{~m}$ as a white solid ( $7.3 \mathrm{mg}, 30 \mu \mathrm{mol} ; 15 \%$ yield).

31: ${ }^{1} \mathrm{H}$ NMR $\left(\mathrm{CDCl}_{3}\right): \delta 7.40-7.31(\mathrm{~m}, 5 \mathrm{H}), 5.54\left(\mathrm{q},{ }^{3} J_{\mathrm{HH}}=6.8 \mathrm{~Hz}, 1 \mathrm{H}\right), 4.49\left(\mathrm{~d},{ }^{2} J_{\mathrm{HH}}=\right.$ $13.7 \mathrm{~Hz}, 1 \mathrm{H}), 3.65\left(\mathrm{~d},{ }^{2} J_{\mathrm{HH}}=13.7 \mathrm{~Hz}, 1 \mathrm{H}\right), 3.29\left(\mathrm{~d},{ }^{2} J_{\mathrm{HH}}=11.5 \mathrm{~Hz}, 1 \mathrm{H}\right), 3.20\left(\mathrm{td}, J_{\mathrm{HH}}=\right.$ 
$10.0 \mathrm{~Hz}$ and $\left.{ }^{3} J_{\mathrm{HH}}=5.0 \mathrm{~Hz}, 1 \mathrm{H}\right), 2.77\left(\mathrm{~d},{ }^{2} J_{\mathrm{HH}}=14.1 \mathrm{~Hz}, 1 \mathrm{H}\right), 2.66\left(\mathrm{q}, J_{\mathrm{HH}}=8.1 \mathrm{~Hz}, 1 \mathrm{H}\right)$, $2.54\left(\mathrm{ddd},{ }^{2} J_{\mathrm{HH}}=16.5 \mathrm{~Hz}\right.$ and ${ }^{3} J_{\mathrm{HH}}=8.9$ and $\left.5.0 \mathrm{~Hz}, 1 \mathrm{H}\right), 2.44-2.37(\mathrm{~m}, 1 \mathrm{H}), 2.28\left(\mathrm{t}, J_{\mathrm{HH}}\right.$ $=12.9 \mathrm{~Hz}, 1 \mathrm{H}), 1.61\left(\mathrm{~d},{ }^{3} J_{\mathrm{HH}}=6.8 \mathrm{~Hz}, 3 \mathrm{H}\right) .{ }^{13} \mathrm{C} \mathrm{NMR}\left(\mathrm{CDCl}_{3}\right): \delta 169.8,140.5,129.5$, 129.0, 128.4, 127.7, 121.0, 71.1, 48.7, 48.6, 36.0, 30.6, 13.0. HRMS (ESI) calcd for $\mathrm{C}_{15} \mathrm{H}_{19} \mathrm{~N}_{2} \mathrm{O}\left(\mathrm{M}+\mathrm{H}^{+}\right)$243.1492, found 243.1482.

3m: ${ }^{1} \mathrm{H}$ NMR $\left(\mathrm{CDCl}_{3}\right): \delta 7.39-7.31(\mathrm{~m}, 5 \mathrm{H}), 4.97(\mathrm{~s}, 1 \mathrm{H}), 4.87\left(\mathrm{q},{ }^{3} J_{\mathrm{HH}}=6.8 \mathrm{~Hz}, 1 \mathrm{H}\right)$, $4.82(\mathrm{~s}, 1 \mathrm{H}), 3.35\left(\mathrm{dd},{ }^{2} J_{\mathrm{HH}}=11.8 \mathrm{~Hz}\right.$ and $\left.{ }^{3} J_{\mathrm{HH}}=3.0 \mathrm{~Hz}, 1 \mathrm{H}\right), 3.18\left(\mathrm{td}, J_{\mathrm{HH}}=9.9 \mathrm{~Hz}\right.$ and $\left.{ }^{3} J_{\mathrm{HH}}=4.8 \mathrm{~Hz}, 1 \mathrm{H}\right), 2.76\left(\mathrm{dd},{ }^{2} J_{\mathrm{HH}}=14.1 \mathrm{~Hz}\right.$ and $\left.{ }^{3} J_{\mathrm{HH}}=12.2 \mathrm{~Hz}, 1 \mathrm{H}\right), 2.61\left(\mathrm{q}, J_{\mathrm{HH}}=9.5\right.$ $\mathrm{Hz}, 1 \mathrm{H}), 2.54\left(\mathrm{ddd},{ }^{2} J_{\mathrm{HH}}=16.1 \mathrm{~Hz}\right.$ and ${ }^{3} J_{\mathrm{HH}}=8.8$ and $\left.4.9 \mathrm{~Hz}, 1 \mathrm{H}\right), 2.42-2.33(\mathrm{~m}, 2 \mathrm{H})$, $1.46\left(\mathrm{~d},{ }^{3} \mathrm{~J}_{\mathrm{HH}}=6.8 \mathrm{~Hz}, 3 \mathrm{H}\right) .{ }^{13} \mathrm{C} \mathrm{NMR}\left(\mathrm{CDCl}_{3}\right): \delta 169.4,143.2,140.5,129.0,128.4,127.6$, $110.9,72.1,53.0,48.6,39.2,31.0,17.9$. HRMS (ESI) calcd for $\mathrm{C}_{15} \mathrm{H}_{19} \mathrm{~N}_{2} \mathrm{O}\left(\mathrm{M}+\mathrm{H}^{+}\right)$ 243.1492, found 243.1483 .

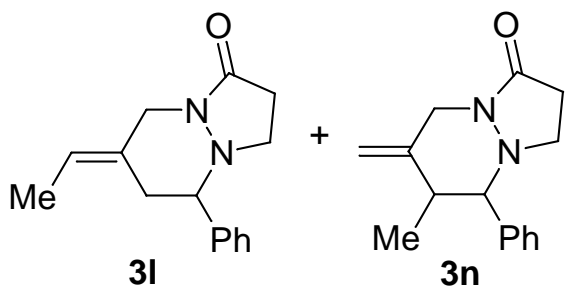

Procedure for Equation 5.

A solution of $\mathrm{Pd}\left(\mathrm{PPh}_{3}\right)_{4}(18.5 \mathrm{mg}, 16.0 \mu \mathrm{mol})$, (2-(acetoxymethyl)-1-buten-3yl)trimethylsilane $5(80.1 \mathrm{mg}, 0.400 \mathrm{mmol})$, and azomethine imine $\mathbf{2 a}(34.8 \mathrm{mg}, 0.200$ $\mathrm{mmol})$ in $\mathrm{CH}_{2} \mathrm{Cl}_{2}(1.0 \mathrm{~mL})$ was stirred for $72 \mathrm{~h}$ at $40{ }^{\circ} \mathrm{C}$, and the reaction mixture was directly passed through a pad of silica gel with EtOAc. After removing the solvent under vacuum, the residue was purified by silica gel preparative TLC with EtOAc/hexane $=1 / 1$ to afford a mixture of compounds $3 \mathbf{l}-\mathbf{3 n}$ as a colorless oil (32.1 $\mathrm{mg}, 0.132 \mathrm{mmol} ; 66 \%$ yield).

3n (mixture of cis/trans 46/54): ${ }^{1} \mathrm{H}$ NMR $\left(\mathrm{CDCl}_{3}\right): \delta 7.39-7.27(\mathrm{~m}, 5 \mathrm{H}), 5.10$ (s, $0.54 \mathrm{H}), 5.00$ (bs, $0.46 \mathrm{H}), 4.924(\mathrm{~s}, 0.54 \mathrm{H}), 4.918(\mathrm{~s}, 0.46 \mathrm{H}), 4.63\left(\mathrm{~d},{ }^{2} \mathrm{~J}_{\mathrm{HH}}=13.7 \mathrm{~Hz}\right.$, $0.54 \mathrm{H}), 4.47(\mathrm{bs}, 0.46 \mathrm{H}), 3.83(\mathrm{bs}, 0.46 \mathrm{H}), 3.72\left(\mathrm{~d},{ }^{2} J_{\mathrm{HH}}=13.7 \mathrm{~Hz}, 0.54 \mathrm{H}\right), 3.65$ (bs, 0.46 $\mathrm{H}), 3.38(\mathrm{bs}, 0.46 \mathrm{H}), 3.07\left(\mathrm{td}, J_{\mathrm{HH}}=10.0 \mathrm{~Hz}\right.$ and $\left.{ }^{3} J_{\mathrm{HH}}=4.6 \mathrm{~Hz}, 0.54 \mathrm{H}\right), 2.94\left(\mathrm{~d},{ }^{3} J_{\mathrm{HH}}=\right.$ $10.3 \mathrm{~Hz}, 0.54 \mathrm{H}), 2.63-2.35(\mathrm{~m}, 4 \mathrm{H}), 0.99\left(\mathrm{~d},{ }^{3} J_{\mathrm{HH}}=6.0 \mathrm{~Hz}, 1.38 \mathrm{H}\right), 0.80\left(\mathrm{~d},{ }^{3} J_{\mathrm{HH}}=6.6\right.$ $\mathrm{Hz}, 1.62 \mathrm{H}) .{ }^{13} \mathrm{C} \mathrm{NMR}\left(\mathrm{CDCl}_{3}\right): \delta 169.8,169.4,143.3,139.0,128.9,128.54,128.52,127.9$, 111.0, 110.1, 78.0, 77.5, 49.0, 48.6, 43.9, 41.7, 30.7, 30.6, 14.0, 13.5. Anal. Calcd for 


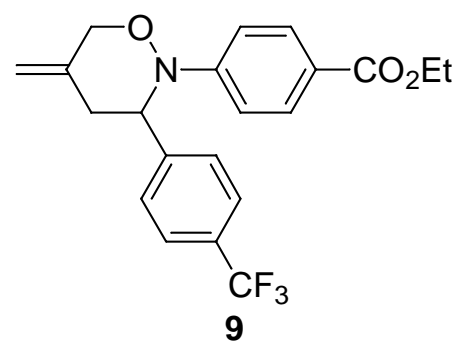

Procedure for Equation 6.

A solution of $\mathrm{Pd}\left(\mathrm{PPh}_{3}\right)_{4}(11.6 \mathrm{mg}, 10.0 \mu \mathrm{mol})$, (2-(acetoxymethyl)-2propenyl)trimethylsilane 1 ( $46.6 \mathrm{mg}, 0.25 \mathrm{mmol})$, and nitrone $8(33.8 \mathrm{mg}, 0.10 \mathrm{mmol}$ ) in $\mathrm{CH}_{2} \mathrm{Cl}_{2}(0.50 \mathrm{~mL})$ was stirred for $43 \mathrm{~h}$ at $40{ }^{\circ} \mathrm{C}$, and the reaction mixture was directly passed through a pad of silica gel with EtOAc. After removing the solvent under vacuum, the residue was purified by silica gel preparative TLC with EtOAc/hexane $=1 / 4.5$ to afford 9 as a colorless oil ( $35.7 \mathrm{mg}, 91.2 \mu \mathrm{mol} ; 91 \%$ yield).

${ }^{1} \mathrm{H} \mathrm{NMR}\left(\mathrm{CDCl}_{3}\right): \delta 7.88\left(\mathrm{~d},{ }^{3} J_{\mathrm{HH}}=8.9 \mathrm{~Hz}, 2 \mathrm{H}\right), 7.51\left(\mathrm{~d},{ }^{3} J_{\mathrm{HH}}=8.8 \mathrm{~Hz}, 2 \mathrm{H}\right), 7.49(\mathrm{~d}$, $\left.{ }^{3} J_{\mathrm{HH}}=8.8 \mathrm{~Hz}, 2 \mathrm{H}\right), 6.94\left(\mathrm{~d},{ }^{3} J_{\mathrm{HH}}=8.9 \mathrm{~Hz}, 2 \mathrm{H}\right), 5.04\left(\mathrm{dd},{ }^{3} J_{\mathrm{HH}}=6.0\right.$ and $\left.4.7 \mathrm{~Hz}, 1 \mathrm{H}\right)$, $4.99(\mathrm{~s}, 1 \mathrm{H}), 4.94(\mathrm{~s}, 1 \mathrm{H}), 4.68\left(\mathrm{~d},{ }^{2} J_{\mathrm{HH}}=12.8 \mathrm{~Hz}, 1 \mathrm{H}\right), 4.57\left(\mathrm{~d},{ }^{2} J_{\mathrm{HH}}=12.7 \mathrm{~Hz}, 1 \mathrm{H}\right), 4.31$ $\left(\mathrm{q},{ }^{3} J_{\mathrm{HH}}=7.1 \mathrm{~Hz}, 2 \mathrm{H}\right), 3.09\left(\mathrm{dd},{ }^{2} J_{\mathrm{HH}}=14.0 \mathrm{~Hz}\right.$ and $\left.{ }^{3} J_{\mathrm{HH}}=6.3 \mathrm{~Hz}, 1 \mathrm{H}\right), 2.72\left(\mathrm{dd},{ }^{2} J_{\mathrm{HH}}=\right.$ $13.9 \mathrm{~Hz}$ and $\left.{ }^{3} J_{\mathrm{HH}}=4.4 \mathrm{~Hz}, 1 \mathrm{H}\right), 1.35\left(\mathrm{t},{ }^{3} J_{\mathrm{HH}}=7.1 \mathrm{~Hz}, 3 \mathrm{H}\right) .{ }^{13} \mathrm{C} \mathrm{NMR}\left(\mathrm{CDCl}_{3}\right): \delta 166.6$, 152.0, 144.1, 139.3, 131.0, $129.8\left(\mathrm{q},{ }^{2} J_{\mathrm{CF}}=32.6 \mathrm{~Hz}\right), 128.3,125.5\left(\mathrm{q},{ }^{3} J_{\mathrm{CF}}=3.6 \mathrm{~Hz}\right), 124.3$ $\left(\mathrm{q},{ }^{1} J_{\mathrm{CF}}=272 \mathrm{~Hz}\right), 123.1,114.4,111.8,74.3,63.3,60.7,37.5,14.6$. HRMS (ESI) calcd for $\mathrm{C}_{21} \mathrm{H}_{21} \mathrm{~F}_{3} \mathrm{NO}_{3}\left(\mathrm{M}+\mathrm{H}^{+}\right)$392.1468, found 392.1463 .

\section{X-ray Crystal Structure of 3k}

\section{Data Collection}

A colorless $\mathrm{Et}_{2} \mathrm{O}$ solution of $\mathbf{3 k}$ was prepared. Crystals suitable for $\mathrm{X}$-ray analysis were obtained by slow evaporation of $\mathrm{Et}_{2} \mathrm{O}$ at room temperature.

A colorless prism crystal of $\mathrm{C}_{15} \mathrm{H}_{18} \mathrm{~N}_{2} \mathrm{O}$ having approximate dimensions of $0.52 \mathrm{x}$ $0.30 \times 0.10 \mathrm{~mm}$ was mounted on a glass fiber. All measurements were made on a Rigaku RAXIS RAPID imaging plate area detector with graphite monochromated Mo-K $\alpha$ radiation. 
Indexing was performed from 3 oscillations that were exposed for 30 seconds. The crystal-to-detector distance was $127.40 \mathrm{~mm}$.

Cell constants and an orientation matrix for data collection corresponded to a primitive triclinic cell with dimensions:

$$
\begin{aligned}
& a=7.076(5) \AA \quad \alpha=77.94(3)^{\circ} \\
& b=7.693(5) \AA \quad \beta=84.96(3)^{\circ} \\
& c=12.82(1) \AA \quad \gamma=75.35(3)^{\circ} \\
& V=659.7(8) \AA^{3}
\end{aligned}
$$

For $Z=2$ and F.W. $=242.32$, the calculated density is $1.22 \mathrm{~g} / \mathrm{cm}^{3}$. Based on a statistical analysis of intensity distribution, and the successful solution and refinement of the structure, the space group was determined to be:

$$
\mathrm{P}-1 \text { (\#2) }
$$

The data were collected at a temperature of $-150 \pm 1^{\circ} \mathrm{C}$ to a maximum $2 \theta$ value of $54.9^{\circ}$. A total of 44 oscillation images were collected. A sweep of data was done using $\omega$ scans from 130.0 to $190.0^{\circ}$ in $5.0^{\circ}$ step, at $\chi=45.0^{\circ}$ and $\phi=0.0^{\circ}$. The exposure rate was 110.0 [sec. $/^{\circ}$ ]. A second sweep was performed using $\omega$ scans from 0.0 to $160.0^{\circ}$ in $5.0^{\circ}$ step, at $\chi=45.0^{\circ}$ and $\phi=180.0^{\circ}$. The exposure rate was 110.0 [sec. $/{ }^{\circ}$ ]. The crystal-to-detector distance was $127.40 \mathrm{~mm}$. Readout was performed in the 0.100 mm pixel mode.

\section{Data Reduction}

A total of 3001 reflections was collected.

The linear absorption coefficient, $\mu$, for Mo-K $\alpha$ radiation is $0.8 \mathrm{~cm}^{-1}$. The data were corrected for Lorentz and polarization effects.

\section{Structure Solution and Refinement}

The structure was solved by direct methods ${ }^{7}$ and expanded using Fourier techniques. ${ }^{8}$ The non-hydrogen atoms were refined anisotropically. Hydrogen atoms

7 SIR92: Altomare, A.; Cascarano, G.; Giacovazzo, C.; Guagliardi, A.; Burla, M.; Polidori, G.; Camalli, M. J. Appl. Cryst. 1994, 27, 435. 
were refined using the riding model. The final cycle of full-matrix least-squares

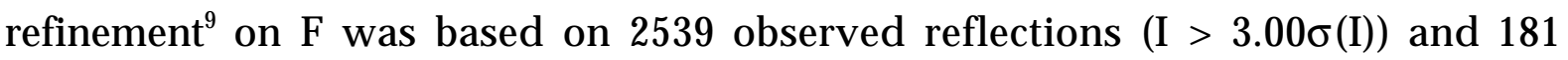
variable parameters and converged (largest parameter shift was 0.00 times its esd) with unweighted and weighted agreement factors of:

$$
\begin{gathered}
\mathrm{R}=\Sigma|| \mathrm{Fo}_{\mathrm{O}}|-| \mathrm{FC}_{\mathrm{C}}|| / \Sigma\left|\mathrm{Fo}_{\mathrm{O}}\right|=0.046 \\
\mathrm{R}_{\mathrm{W}}=\left[\Sigma \mathrm{W}\left(\left|\mathrm{Fo}_{\mathrm{O}}\right|-\left|\mathrm{FC}_{\mathrm{C}}\right|\right)^{2} / \Sigma \mathrm{W} \mathrm{Fo}^{2}\right]^{1 / 2}=0.064
\end{gathered}
$$

The standard deviation of an observation of unit weight ${ }^{10}$ was 1.34. A Sheldrick weighting scheme was used. Plots of $\Sigma \mathrm{w}\left(|\mathrm{Fo}|-\left|\mathrm{FC}_{\mathrm{C}}\right|\right)^{2}$ versus $|\mathrm{Fo}|$, reflection order in data collection, $\sin \theta / \lambda$ and various classes of indices showed no unusual trends. The maximum and minimum peaks on the final difference Fourier map corresponded to 0.22 and $-0.39 \mathrm{e}^{-} / \AA^{3}$, respectively.

Neutral atom scattering factors were taken from Cromer and Waber. ${ }^{11}$ Anomalous dispersion effects were included in Fcalc, ${ }^{12}$ the values for $\Delta \mathrm{f}^{\prime}$ and $\Delta \mathrm{f}^{\prime \prime}$ were those of Creagh and McAuley. ${ }^{13}$ The values for the mass attenuation coefficients are those of Creagh and Hubbell. ${ }^{14}$ All calculations were performed using the CrystalStructure ${ }^{15,16}$ crystallographic software package.

${ }^{8}$ DIRDIF99: Beurskens, P. T.; Admiraal, G.; Beurskens, G.; Bosman, W. P.; de Gelder, R.; Israel, R; Smits, J. M. M. The DIRDIF-99 program system, Technical Report of the Crystallography Laboratory, University of Nijmegen, The Netherlands (1999).

${ }^{9}$ Least Squares function minimized: (SHELXL97) $\Sigma w\left(\left|\mathrm{~F}_{\mathrm{O}}\right|-\left|\mathrm{F}_{\mathrm{C}}\right|\right)^{2} \quad$ where $\mathrm{w}=$ Least Squares weights.

${ }^{10}$ Standard deviation of an observation of unit weight:

$$
\left[\Sigma w\left(\left|\mathrm{~F}_{\mathrm{O}}\right|-\left|\mathrm{F}_{\mathrm{C}}\right|\right)^{2} /\left(\mathrm{N}_{\mathrm{O}}-\mathrm{N}_{\mathrm{V}}\right)\right]^{1 / 2}
$$

where: $\mathrm{N}_{\mathrm{O}}=$ number of observations, $\mathrm{N}_{\mathrm{V}}=$ number of variables

${ }^{11}$ Cromer, D. T.; Waber, J. T. "International Tables for X-ray Crystallography", Vol. IV, The Kynoch Press, Birmingham, England, Table 2.2 A (1974).

${ }^{12}$ Ibers, J. A.; Hamilton, W. C. Acta Crystallogr. 1964, 17, 781.

${ }^{13}$ Creagh, D. C.; McAuley, W. J . "International Tables for Crystallography", Vol C, (Wilson, A. J. C., ed.), Kluwer Academic Publishers, Boston, Table 4.2.6.8, pages 219-222 (1992).

${ }^{14}$ Creagh, D. C.; Hubbell, J. H. "International Tables for Crystallography", Vol C, (Wilson, A. J. C., ed.), Kluwer Academic Publishers, Boston, Table 4.2.4.3, pages 200-206 (1992).

${ }^{15}$ CrystalStructure 3.6.0: Crystal Structure Analysis Package, Rigaku and Rigaku/MSC (2000-2004). 9009 New Trails Dr. The Woodlands TX 77381 USA.

${ }^{16}$ CRYSTALS Issue 10: Watkin, D. J.; Prout, C. K.; Carruthers, J. R.; Betteridge, P. W. Chemical Crystallography Laboratory, Oxford, UK. (1996). 
The crystal structure has been deposited at the Cambridge Crystallographic Data Centre (deposition number: CCDC 297116). The data can be obtained free of charge via the Internet at www.ccdc.cam.ac.uk/conts/retrieving.html.

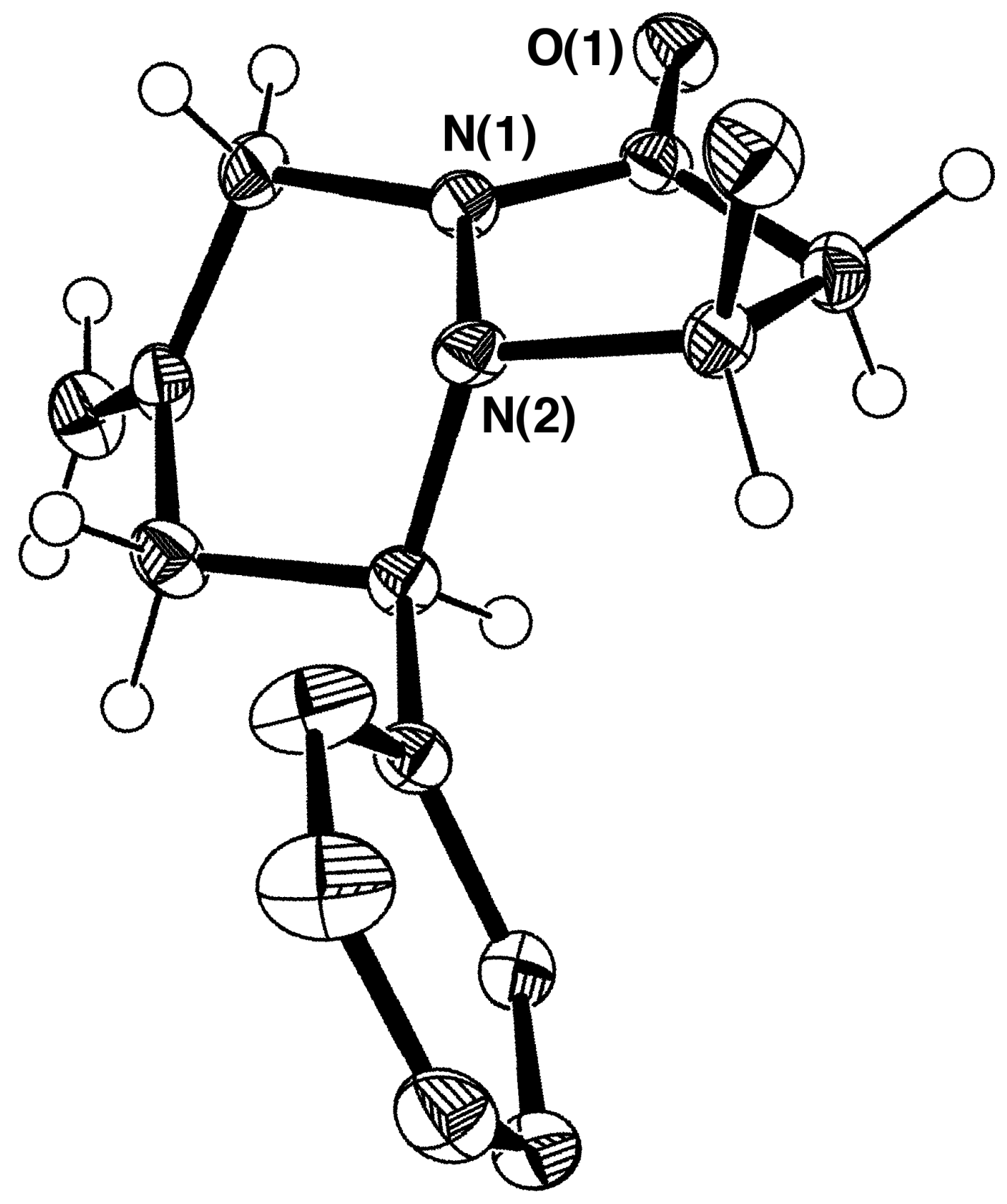


Experimental Details

\section{A. Crystal Data}

Empirical Formula

$\mathrm{C}_{15} \mathrm{H}_{18} \mathrm{~N}_{2} \mathrm{O}$

Formula Weight

242.32

Crystal Color, Habit

colorless, prism

Crystal Dimensions

$0.52 \times 0.30 \times 0.10 \mathrm{~mm}$

Crystal System

triclinic

Lattice Type

Primitive

Indexing Images

3 oscillations @ 30.0 seconds

Detector Position

$127.40 \mathrm{~mm}$

Pixel Size

$0.100 \mathrm{~mm}$

Lattice Parameters

$$
\begin{aligned}
& \mathrm{a}=7.076(5) \AA \\
& \mathrm{b}=7.693(5) \AA \\
& \mathrm{c}=12.82(1) \AA \\
& \alpha=77.94(3)^{\circ} \\
& \beta=84.96(3)^{\circ} \\
& \gamma=75.35(3)^{\circ} \\
& \mathrm{V}=659.7(8) \AA^{3}
\end{aligned}
$$

Space Group

P-1 (\#2)

$Z$ value

2

D calc

$1.220 \mathrm{~g} / \mathrm{cm}^{3}$

F000

260.00

$\mu(\operatorname{MoK} \alpha)$

$0.77 \mathrm{~cm}^{-1}$ 


\section{B. Intensity Measurements}

Diffractometer

Radiation

Detector Aperture

Data Images

$\omega$ oscillation Range $((\chi=45.0, \phi=30.0)$

Exposure Rate

$\omega$ oscillation Range $(\chi=45.0, \phi=180.0)$

Exposure Rate

Detector Position

Pixel Size

$2 \theta_{\max }$

No. of Reflections Measured

Corrections
Rigaku RAXIS-RAPID

$\operatorname{MoK} \alpha(\lambda=0.71075 \AA)$

graphite monochromated

$280 \mathrm{~mm} \times 256 \mathrm{~mm}$

44 exposures

$130.0-190.0^{\circ}$

$110.0 \mathrm{sec} .{ }^{\circ}$

$0.0-160.0^{\circ}$

$110.0 \mathrm{sec} . /^{\circ}$

$127.40 \mathrm{~mm}$

$0.100 \mathrm{~mm}$

$54.9^{\circ}$

Total: 3001

Lorentz-polarization 


\section{Structure Solution and Refinement}

Structure Solution

Refinement

Function Minimized

Least Squares Weights

$2 \theta_{\max }$ cutoff

Anomalous Dispersion

No. Observations $(\mathrm{I}>3.00 \sigma(\mathrm{I}))$

No. Variables

Reflection/Parameter Ratio

Residuals: R (I>3.00б(I))

Residuals: Rw (I>3.00 $\sigma(\mathrm{I}))$

Goodness of Fit Indicator

Max Shift/Error in Final Cycle

Maximum peak in Final Diff. Map

Minimum peak in Final Diff. Map
Direct Methods (SIR92)

Full-matrix least-squares on $\mathrm{F}$

$\Sigma_{\mathrm{W}}\left(\left|\mathrm{Fo}_{\mathrm{O}}\right|-\left|\mathrm{FC}_{\mathrm{C}}\right|\right)^{2}$

$\mathrm{w}=1 /\left[0.0010 \mathrm{Fo}^{2}+3.0000 \sigma\left(\mathrm{Fo}^{2}\right)+\right.$ 0.5000]

$0.0^{\circ}$

All non-hydrogen atoms

2539

181

14.03

0.046

0.064

1.340

0.000

$0.22 \mathrm{e}^{-} / \AA^{3}$

$-0.39 \mathrm{e}^{-} / \AA^{3}$ 